\title{
On the stability of thermally stratified \\ plane Poiseuille flow
}

\section{Summary}

The stability of thermally stratified plane Polseuille flow is considered with respect to small disturbances. The interaction between the usual Tollmien Schlichting type of instability and the thermal type of (in)stability is analysed for various values of the Prandtl number. The problem is solved as an eigenvalue problem. The most important features from the results are that the critical Rayleigh number is found to be nearly linearly dependent of the Prandt n number, and that a critical Reynolds number always exists, no matter how great the fluid is stabilized by a linear temperature profile. 


\section{Introduction.}

Great attention has for many years been paid to the stability of shear flow. The problem is basic in studying the transition from laminar to turbulent flow. Additionally, the equations involved define an interesting mathematical problem.

The non-stratified problem was first successfully attached by Heisenberg [1]. His ideas are later applied and extended by several investigators. Most notably are the stability analysis by Tolimien [2] and Schlichting [3] for boundary layer flow and by Lin [4] for plane Poiseuille flow.

Vertical density stratification further complicates the stability problem. Koppel [5] considered the thermally stratified problem and obtained asymptotic solutions of the perturbation equation. Utilizing Koppel's solutions Gage and Reid [6] analysed the stability of plane Poiseuile flow with the simplifying assumption of unit Prandtl number. Hughes and Reid [7] also considered Koppel's solutions in studying the stability of spiral flow between rotating cylinders. For this problem the governing equations turn out to be mathematically identical to those analysed in [6]. However, in physical fluids the Prandtl number varies from about 0.02 (mercury) to $10^{4}$ (silicon oil), and this variation obviously affects the stability problem. This has motivated the present work, in which we consider thermally stratified Poiseuile flow with arbitrary Prandt number.

The so-called inviscid equation is solved by a two-parameter expansion after the horisontal wave number $k$ and a certain stability parameter s defined by $(3.3)$. This procedure leads to some simplification in the solution of the 
eigenvalue problem compared to the more traditional approach, in which the solutions of the inviscid equation have been based on a power series expansion after the vertical coordinate.

2. The governing equations.

A thermally stratified steady shear flow is considered, which is bounded by two horisontal perfect conductive rigid boundaries. We choose a Cartesian coorainate system ( $\mathrm{x}, \mathrm{y}, \mathrm{z})$ with unit vectors $(\vec{I}, \vec{j}, \vec{k})$, such that the $x$-axis is pointing downstream. The boundaries are at $z= \pm h$.

In deriving the linearized disturbance equations the usual Boussinesq approximation is used. The equation of fluid motion, the continuity equation, the heat equation and the equation of state may then be written

$$
\begin{aligned}
& \rho_{0}\left(\frac{\partial}{\partial t}+U \frac{\partial}{\partial x}-v \nabla^{2}\right) \vec{v}+\rho_{0} w U \cdot \vec{I}=-\nabla p-\rho g \vec{k} \\
& \nabla \cdot \vec{v}=0 \\
& \left(\frac{\partial}{\partial t}+U \frac{\partial}{\partial x}-k \nabla^{2}\right) \theta=\beta w \\
& \rho=\rho_{0}\left(1-\alpha\left(T-T_{0}+\theta\right)\right)
\end{aligned}
$$

Here $U$ and $T$ denote basic velocity and temperature. They are assumed to have the forms

$$
\begin{aligned}
& U=U_{*}\left(1-\left(\frac{z}{h}\right)^{2}\right) \\
& T=T_{1}-\beta(z+h) ; \quad \beta=\frac{T_{1}-T_{2}}{2 h}
\end{aligned}
$$


$\mathrm{T}_{1}$ and $\mathrm{T}_{2}$ are the temperatures of upper and lower boundary, respectively. $T_{0}$ is a standard temperature, and $\theta$ the departure from the basic temperature. Furthermore, $\vec{v}=u \vec{i}+v \vec{j}+w \vec{k}$ denotes the velocity of disturbance, $\rho$ the density, $\rho_{0}$ a standard density, $p$ the pressure, and $g$ the acceleration of gravity. $\alpha, k$ and $v$ are the coefficients of volume expansion, thermal conductivity and kinematic viscosity, respectively.

It is convenient to non-dimensionalize the equations in terms of a characteristic length $L_{*}=h$, a characterist1c velocity $U_{*}$ and a characteristic temperature $T_{*}=\frac{1}{2}\left|T_{1}-T_{2}\right|$. The Reynolds and the Rayleigh number are defined in the usual way by

$$
\mathrm{R}=\frac{\mathrm{U}_{*} \mathrm{~h}}{\nu} \quad \text { and } \quad \mathrm{Ra}=\frac{\alpha \mathrm{g}(2 \mathrm{~h})^{3}(2 \mathrm{~T} *)}{\mathrm{K \nu}}
$$

Applying Fouriers theorem the disturbances may be written in the form

$$
f(z) \exp \{i k(x-c t)+i l y\},
$$

where $f(z)$ is an amplitude function, $c$ the complex phase velocity, and $k$ and $l$ the wave numbers. Elimination of $u, v, \theta, p$ and $\rho$ between (2.1) - (2.4) gives an equation for the amplitude $w(z)$ of the vertical velocity component $w(x, y, z, t)$

$$
D \mathscr{L}_{\mathrm{w}}+\frac{1}{16} \operatorname{Ra} \mathrm{a}^{2} \mathrm{w}=0
$$

Here

$$
\begin{aligned}
& \mathscr{D}=i k R P(U-c)-\left(D^{2}-a^{2}\right), \\
& \mathscr{L}=\left(i k R(U-c)-\left(D^{2}-a^{2}\right)\right)\left(D^{2}-a^{2}\right)-i k R U^{\prime \prime}, \\
& D=\frac{d}{d z}, \\
& a^{2}=k^{2}+1^{2},
\end{aligned}
$$

and $\quad P=\frac{\nu}{K}$ is the Prandt $I$ number. 
The boundary conditions applying are

$$
w=D w=\mathscr{L} w=0 \quad \text { at } z= \pm 1
$$

In examining the stability of the flow we shall only consider two-dimensional perturbations (independent of y). From the solution of this two-dimensional problem it is possible to deduce the solution of the general three-dimensional problem by using a generaIlzation of Squire's transformations, given in [6]. These transformations propose that the present two-dimensional mode is the most undstable one for $\mathrm{Ra}$ less the critical Rayleigh number without shear (1708). Further, for Ra greater than 1708 the mode independent of $x$ is the critical one, and the stability problem is solved by the ordinary Bénard problem. Primarily, we take interest in the most critical mode, and the general three-dimensional solution will therefore not be obtained.

\section{Asymptotic solutions.}

It is well known for stable or weak unstable stratification, that instability first occur, as in the non-stratified case, for values of the combined parameter $k R$ much greater than one. This fact is basic in finding asymptotic solutions of (2.5). One natural way to obtain such solutions is to write the solutions as the formal expansion

$$
w=w_{0}+\frac{1}{k R} w_{1}+\cdots \cdots
$$

so that the initial approximation $w_{0}$ is a solution of the inviscid equation 


$$
\left(D^{2}-\frac{U^{\prime \prime}}{U-C}\right) w-k^{2} w-\frac{S}{(U-C)^{2}} w=0
$$

Here

$$
s=\frac{\frac{1}{16} R a}{R^{2} P}
$$

is an introduced stability parameter, closely connected with the Richardsons number. When $c \neq U$, the solutions of (3.2) may conveniently be written as uniformly convergent series of $s$ and $k^{2}$ in the form

$$
w_{1,2}=\sum_{n=0}^{\infty} \sum_{m=0}^{\infty} k^{2 n} s^{m} w_{n, m}^{1,2}
$$

Here

$$
\begin{aligned}
& w_{0,0}^{1}=(U-c) \\
& w_{0,0}^{2}=(U-c) \int_{-1}^{z} \frac{d z}{(U-c)^{2}} \\
& w_{n, m}^{1,2}=(U-c) \int_{-1}^{z} \frac{d z}{(U-c)^{2}} \int_{-1}^{z} d z(U-c)\left\{w_{n-1, m^{1}, 2}+\frac{1}{(U-c)^{2}} w_{n, m-1}^{1,2}\right\} .
\end{aligned}
$$

This form of the solutions differ from that given previously in related problems. (3.4) is suitable for the purpose of finding the eigenvalue c. For $z=-1 w_{1,2}$ are easily obtained. For $z=0$ it will turn out that it is sufficient to take into account the four terms with $(n, m) \leq(1,1)$. However, to assure the convergence the 16 terms with $(n, m) \leq(3,3)$ are used in the present calculations.

To obtain the other solutions of (2.5) it is necessary to take Into consideration the effect of viscosity and conductivity. We apply a transformation first proposed by He1senberg [1]. Let

$$
\varepsilon=(k R)^{-\frac{1}{3}}, \quad \zeta=\frac{z-z_{c}}{\varepsilon}
$$


and

$$
w(z)=\varphi(\zeta) .
$$

Furthermore, we write

$$
\varphi=\varphi_{0}+\varepsilon \varphi_{1}+
$$

Substituting this into (2.5) the first approximation $\varphi_{0}$ satisfies the equation

$$
\left(d^{2}-1 P U_{c}^{\prime} \zeta\right)\left(d^{2}-i U_{c}^{\prime} \zeta\right) d^{2} \varphi+U_{c}^{\prime 2} P \operatorname{Ri} \varphi=0
$$

Here

$$
\begin{aligned}
& d=\frac{d}{d \zeta} \cdot \\
& R I=-\frac{s}{U_{c}^{2}}
\end{aligned}
$$

is the Ricahrdson number at the level $z=z_{c} \cdot z_{c}$ is defined by $U\left(z_{c}\right)=U_{c}=c . \quad(3.6)$ is the equation considered by KoppeI [5]. His solutions are given in the Appendix $A$.

The solutions $w_{1}$ and $w_{2}$ need some remarks. Since almost every term of the series, which defines $w_{1}$ and $w_{2}$, has logaritmic singularity in $z_{c}$, neither of them can provide uniformly valid asymptotic approximations to any solutions of equation (2.5) in a full complex neighbourhood of $z_{c}$. However, by considering their corresponding "viscous and conductive" solutions, it is shown in the Appendix $C$ that $w_{1}$ and $w_{2}$ do provide valid asymptotic solutions in the sector

$$
-\frac{7 \pi}{6}<\arg \left(z-z_{c}\right)<\frac{\pi}{6}
$$

of the complex $z$-plane. 
4. The eigenvalue problem.

The general solution of $(2.5)$ is represented in the form

$$
w=\sum_{i=1}^{6} c_{i} w_{i}
$$

where $\mathrm{w}_{4}, \mathrm{w}_{6}$ and $\mathrm{w}_{3}, \mathrm{w}_{5}$ are given by Koppel's solutions $\varphi^{(1)}$ and $\varphi^{(3)}$, respectively. Since (2.5) and (2.6) are symmetrical in $z$, the odd and the even part of (4.1) can be applied separately. Only the even mode will be considered, because this mode is the most unstable one. For an even solution the conditions

$$
w^{\prime}=w^{\prime \prime \prime}=w^{(5)}=0 \text { at } z=0
$$

must be satisfied. Thus the boundary conditions (2.6) for $z=1$ can be replaced by $(4.2)$.

Appendix $B$ and $D$ shows that $w_{4}$ and $w_{6}$ are eksponentially great for $z=0$. Consequently, these solutions must be rejected, because we expect that viscous and conductive effects are negligible in the central part of the channel. Furthermore, $w_{3}$ and $w_{5}$ are exponentially small for $z=0$. Thus, if we let

$$
W=-\frac{W_{2}^{\prime}(0)}{W_{1}^{1}(0)} w_{1}+w_{2}
$$

then, since $U(z)$ is an even function, $W$ automatically satisfies the conditions (4.2). The boundary conditions (2.6) for $z=-1$ then give

$$
\left|\begin{array}{rrr}
\left.W^{(}-1\right) & W_{3}(-1) & W_{5}(-1) \\
W^{9}(-1) & W_{3}^{\prime}(-1) & W_{5}^{1}(-1) \\
\mathscr{L} W^{\prime}(-1) & \mathscr{L} W_{3}(-1) & \mathscr{L} W_{5}(-1)
\end{array}\right|=0
$$


From (3.2)

$$
\mathscr{L}_{W}(-1)=\frac{1 k R s}{c} W(-1)(1+O(\varepsilon))
$$

and from $(3.4)$

$$
\begin{array}{ll}
w_{1}(-1)=-c, & w_{2}(-1)=0 \\
w_{1}^{\prime}(-1)=U^{\prime}(-1)=2, & w_{2}^{\prime}(-1)=-\frac{1}{c} .
\end{array}
$$

Evaluation of the determinant, using the above expressions, gives

$$
\frac{w_{2}^{\prime}(0)}{w_{1}^{\prime}(0)}=
$$

$\frac{\frac{1}{c}\left(\frac{\mathscr{L} W_{3}(-1)}{W_{3}(-1)}-\frac{\mathscr{L} W_{5}(-1)}{W_{5}(-1)}\right)}{c\left(\frac{W_{3}^{\prime}(-1)}{W_{3}(-1)} \frac{\mathscr{L} W_{5}(-1)}{W_{5}(-1)}-\frac{W_{5}^{\prime}(-1)}{W_{5}(-1)} \frac{\mathscr{L} W_{3}(-1)}{W_{3}(-1)}\right)+2\left(\frac{\mathscr{L} W_{5}(-1)}{W_{5}(-1)}-\frac{\mathscr{L} W_{3}(-1)}{W_{3}(-1)}\right)+1 k R s\left(\frac{W_{3}^{\prime}(-1)}{W_{3}(-1)}-\frac{W_{5}^{\prime}(-1)}{W_{5}(-1)}\right)}$

This is a complex relation between the eigenvalue $c=c_{r}+i c_{i}$ and the parameters $k, R, R i_{c}, P$ and $s$. Results from the numerical solution of (4.5) in the neutral case, $c_{i}=0$, are given in the figures and the table below. Points inside the neutral curves correspond to unstable conditions.

Figure 4.1 gives $R=R(k)$, and $c=c(k)$ is given in figure 4.2. The results are very little depended on the Prandt number. No effects of the Prandt I number is found when this is larger than 0.7. A comparison shows that present results are in accordance with the results in [6] and [7]. Calculations of (4.5) with the Prandt I number less than 0.3 have not been done, because the computationtime of Koppel's solutions increased rapidly with decreasing $P$. 
TABLE 1. Critical values of Reynolds number, phase velocity and wave number for given Richardsons number and Prandt 1 number.

\begin{tabular}{|c|c|c|c|c|c|c|c|c|c|c|c|c|c|}
\hline & \multicolumn{2}{|c|}{$\mathrm{P}=0.3$} & \multicolumn{2}{|c|}{$\mathrm{P}=0.7$} & \multicolumn{3}{|c|}{$\mathrm{P}=2.0$} & \multicolumn{3}{c|}{$\mathrm{P}=10.0$} \\
\cline { 2 - 10 } & $\mathrm{R}^{1 / 3}$ & $\mathrm{k}^{2}$ & $\mathrm{c}$ & $\mathrm{R}^{1 / 3}$ & $\mathrm{k}^{2}$ & $\mathrm{c}$ & $\mathrm{R}^{1 / 3}$ & $\mathrm{k}^{2}$ & $\mathrm{c}$ & $\mathrm{R}^{1 / 3}$ & $\mathrm{k}^{2}$ & $\mathrm{c}$ \\
\hline 0.04 & 10.2 & 1.3 & 0.40 & 10.3 & 1.25 & 0.40 & 10.3 & 1.25 & 0.40 & 10.2 & 1.25 & 0.40 \\
0.01 & 14.4 & 1.15 & 0.31 & 14.9 & 1.1 & 0.30 & 15.1 & 1.1 & 0.30 & 14.9 & 1.1 & 0.30 \\
0.01 & 20.2 & 1.0 & 0.23 & 21.5 & 0.95 & 0.22 & 21.8 & 0.95 & 0.22 & 21.4 & 0.95 & 0.23 \\
0.02 & 25.3 & 0.91 & 0.194 & 27.6 & 0.87 & 0.183 & 28.1 & 0.87 & 0.182 & 27.6 & 0.88 & 0.185 \\
0.03 & 34.0 & 0.85 & 0.152 & 38.9 & 0.78 & 0.136 & 39.6 & 0.78 & 0.135 & 38.7 & 0.79 & 0.139 \\
0.04 & 51.4 & 0.74 & 0.105 & 65.7 & 0.66 & 0.085 & 67.3 & 0.66 & 0.084 & 64.8 & 0.57 & 0.087 \\
0.05 & 114 & 0.61 & 0.050 & 210 & 0.51 & 0.028 & 219 & 0.51 & 0.028 & 193 & 0.53 & 0.031 \\
0.055 & 280 & 0.53 & 0.021 & $\infty$ & 0.38 & 0 & $\infty$ & 0.38 & 0 & $\infty$ & 0.38 & 0 \\
0.058 & $\infty$ & 0.44 & 0 & & & & & & & & & \\
\hline
\end{tabular}

It may be of some interest to obtain the general nature of the neutral curves associated with the limit $R \rightarrow \infty$. From the definition of the parameter $y$ in the Appendix $D$ follows

$$
R=\frac{y^{3}}{U_{C}^{\prime} k(1-\sqrt{1-c})^{3}}
$$

This relation shows that for $R \rightarrow \infty$, at least one of the three following limits must occur
1) $y \rightarrow \infty$,
2) $k \rightarrow 0$,
3) $\mathrm{c} \rightarrow 0$.

Further, we observe that the right hand side of (4.5) tends toward zero as $\frac{1}{y}$, when $y \rightarrow \infty$, and tends toward infinite as $\frac{1}{c}$, when $c \rightarrow 0$. 
The solution of (4.5) in the case of stable stratification indicates that $\mathrm{c} \rightarrow 0$ and $\mathrm{y}<\infty$, as $\mathrm{R} \rightarrow \infty$ along both branches of the neutral curves. Therefore, we suppose $\mathrm{y}<\infty$ and $\mathrm{c} \rightarrow 0$ in trying to find the limits of $k$.

We introduce the relation

$$
\int_{-1}^{z} \frac{d z}{(U-c)^{2}}=\int_{-1}^{0} \frac{d z}{(U-c)^{2}}+\int_{0}^{z} \frac{d z}{(U-c)^{2}}
$$

Applying this to the first integration of all the terms involved in $\mathrm{w}_{2}$, we obtain

$$
w_{2}^{\prime}(0)=A w_{1}^{\prime}(0)+v
$$

For small values of $c$ we have

$$
A \propto \frac{1}{\mathrm{c}} \quad \text { and } \quad \mathrm{V} \propto \ln \mathrm{c} .
$$

(4.8) introduced into (4.5) then gives

$$
\frac{c v}{w_{1}^{\prime}(0)} \rightarrow \text { const., as } c \rightarrow 0
$$

which implies

$$
w_{1}^{\prime}(0) \rightarrow 0, \text { as } c \rightarrow 0
$$

Since

$$
w_{1}^{\prime}(0) \approx k^{2} \int_{-1}^{0} u^{2} d z+s \int_{-1}^{0} d z
$$

for small values of $c$, we get by (3.7) and integration of (4.9)

$$
\mathrm{k}^{2} \approx \frac{15}{2} \mathrm{Ri}
$$

One may then expect that the two branches are joined as $R$ increase to infinite. Consequently, each neutral curve forms a loop for stable stratification. 
This is not the case for unstable stratification. As $R \rightarrow \infty$ along the upper branch, the solution of (4.4) indicates that $y \rightarrow \infty$. Hence

$$
w_{2}^{\prime}(0)=0
$$

gives the limits of $k$ and $c$. As $R \rightarrow \infty$ along the lower branch, we find that $y<\infty$ and $c \neq 0$. Therefore, by putting $k=0$ in (4.4), the limits of $c$ are given. The Influence of $P$ is found to be insignificant.

TABLE 2. The limits of wave number and phase velocity as the Reynolds number tends toward infinite for given Richardsons number. (u.b. = upper branch, I.b. = lower branch).

\begin{tabular}{|c|c|c|c|c|c|c|c|c|c|}
\hline \multirow{2}{*}{ Ri } & \multicolumn{2}{|c|}{-0.04} & \multicolumn{2}{|c|}{-0.01} & 0.01 & 0.02 & 0.03 & 0.04 & 0.05 \\
\cline { 2 - 9 } & u.b. & $1 . b$. & u.b. & $1 . b$. & & & & & \\
\hline $\mathrm{k}^{2}$ & 0.283 & 0 & 0.074 & 0 & 0.075 & 0.15 & 0.225 & 0.30 & 0.375 \\
\hline $\mathrm{c}$ & 0.138 & 0.182 & 0.039 & 0.045 & 0 & 0 & 0 & 0 & 0 \\
\hline
\end{tabular}

\section{Final remarks.}

According to the results presented, the stability of the flow evidently seems to be only slightly depended on the Prandtl number. The most important reason for this weak influence of $P$ is due to the behaviour of the solution in the central part of the channel. Indeed, the "inner solution" is independent of $\mathrm{P}$, because Ri (or s) is used as our stability parameter. If we had solved the characteristic equation (4.5) with $\mathrm{Ra}=$ const. instead of $\mathrm{Ri}=$ const., we 
could have found a significant influence of $P$. This is seen by the relation

$$
R a=64 R I(1-c) R P
$$

which is given by (3.3) and (3.7). An almost linear dependence of $P$ would occur in the neutral curves.

In figure 5.1 the neutral curves in the $(R, R a)$-plane are sketched. The figure indicates the linear dependence of $P$. From the figure we also see that each value of the Rayleigh number has a corresponding Reynolds number that makes the flow unstable. Therefore, it is impossible to make plane Poisevile flow completely stable by a linear temperature gradient.

Furthermore, from the figure $5 \cdot 1$, about $R^{1 / 3} \approx(5400)^{1 / 3} \approx 17 \cdot 5$ the instability is of the usual Tollmien Schlichting type. For lesser and higher values of $R$ the Tollmien Schlichting mode of instability is stabilized and instabilized, respectively, by the thermal stratification. However, when the stratification is unstable, the stability-question is reduced to the ordinary Bénard problem (see section 2). Hence the instability is of the thermal type for $R<5400$ and the critical Rayleigh number is in the reality $\mathrm{Ra}^{1 / 6} \approx(1708)^{1 / 6} \approx 3 \cdot 5$.

\section{Acknowledgement.}

The author is indebted to Dr. E. Riis for valuable discussions throughout the work with this problem, and to Professor E. Palm for reading the manuscript. 


\section{APPENDIX}

A. The solutions of (3.6).

For solving (3.6) we follow Koppel [5], who gave the solution

$$
\varphi^{(i)}(\zeta)=\int_{\Gamma_{i}} e^{\zeta t} v(t) d t
$$

where

$$
v(t)=t^{-3} \exp \left\{\frac{i}{\sigma U_{C}^{1}}\left(\frac{1}{P}+1\right) t^{3}\right\} \times \mathbb{M}_{k, m}\left\{\frac{i}{3 U_{c}^{1}}\left(\frac{1}{P}-1\right) t^{3}\right\}
$$

Here $M_{k, m}$ is the Whittaker-function $[8, p .6]$ with

$$
k=\frac{1}{3} \quad \text { and } m= \pm \frac{1}{6} \sqrt{1-4 \mathrm{RI}^{*}}
$$

For every choice of the path of integration $\Gamma_{i}$ we get two solutions, $\varphi_{1}^{(i)}$ and $\varphi_{2}^{(1)}$ by using plus and minus in $m$, respectively. $\Gamma$ 's endpoints, $a$ and $b$, must satisfy the equation

$$
\left.e^{\zeta t}\left\{\zeta t^{2} v-\frac{d}{d t}\left(t^{2} v\right)+\frac{i}{U_{c}^{i}}\left(\frac{1}{P}+1\right) t^{4} v\right\}\right|_{a} ^{b}=0
$$

In the present paper we have defined six different paths of integration (see the figure $A 1$ ). At most three of them may give linearly indeperdent solutions. The linear expression

$$
\varphi^{(1)}(\zeta)+\varphi^{(2)}(\zeta)+\varphi(3)(\zeta)+\varphi^{(5)}(\zeta)=0
$$

obviously follows from the figure A1. In addition from (A4), applying Kummer's transformation [8,p.6] and the transformation

$$
\zeta=\zeta e^{ \pm 1 \frac{2 \pi}{3}}
$$

we get

$$
-e^{i 6 m \pi_{\varphi}(1)}(\zeta)+\varphi^{(2)}(\zeta)+\varphi^{(3)}(\zeta)+\varphi^{(6)}(\zeta)=0
$$


and

$$
\varphi^{(1)}(\zeta)+\varphi^{(2)}(\zeta)-e^{i 6 m \pi} \varphi^{(3)}(\zeta)+\varphi^{(4)}(\zeta)=0
$$

B. Asymptotic expansion of (A1).

The definition of the Whittaker-function $w_{k, m}[8, p .6]$ and Kummer's transformation give

$$
\begin{gathered}
M_{k, m}(z)=e^{-i 2 n\left(m+\frac{1}{2}\right) \pi} \Gamma(2 m+1) e^{-i k \pi\left\{\frac{W}{-k, m}\left(z e^{i(2 n-1) \pi}\right)\right.} \\
\Gamma\left(m-k+\frac{1}{2}\right)
\end{gathered}
$$

and

$$
\begin{aligned}
& M_{k, m}(z)=e^{1(-2 n+1)\left(m+\frac{1}{2}\right) \pi} \Gamma(2 m+1) e^{i k \pi}\left\{\frac{W_{2}\left(z e^{i 2 n \pi}\right)}{\Gamma\left(m+k+\frac{1}{2}\right)}\right. \\
& \left.+\frac{e^{i\left(m+\frac{1}{2}\right) \pi_{W}}-k, m\left(z e^{i(2 n+1) \pi}\right)}{\Gamma\left(m-k+\frac{1}{2}\right)}\right\} \\
& \mathrm{n}=0, \pm 1, \cdots
\end{aligned}
$$

It is known that

$$
W_{k, m}(z)=e^{-\frac{z}{2}} z^{k}\left(1+O\left(\frac{1}{z}\right)\right) ;|\arg z|<\frac{3 \pi}{2}
$$

The above expression substituted into (B1) and (B2) gives asymptotic formulae for $M_{k, m}$ valid for $\left(-\frac{1}{2}-2 n\right) \pi<\arg z<\left(\frac{3}{2}-2 n\right) \pi$ and $\left(-\frac{3}{2}-2 n\right) \pi<\arg z<\left(\frac{1}{2}-2 n\right) \pi$, respectively. Th1s asymptotic behaviour of $M_{k, m}$ implies that 


$$
e^{\zeta t} v(t) \sim A_{1} t^{-2} \exp \left\{\zeta t+\frac{i}{3 U_{c}^{T}} t^{3}\right\}+A_{2} t^{-4} \exp \left\{\zeta t+\frac{i}{3 U_{c}^{T} P} t^{3}\right\}
$$

where $A_{1}$ and $A_{2}$ depend on $m$ and the choice of argument.

The asymptotic expansion of $\varphi^{(1)}, \varphi^{(2)}$ and $\varphi^{(3)}$ can then be obtained by the usual method of steepest descent, and we get

$$
\varphi^{(1,2,3)}(\zeta) \sim B_{1}(B \zeta)^{-\frac{5}{4}} \exp \left\{\frac{2}{3}(B \zeta)^{\frac{3}{2}} \gamma\right\}+B_{2}(\alpha \zeta)^{-\frac{9}{4}} \exp \left\{\frac{2}{3}(\alpha \zeta)^{\frac{3}{2}} \gamma\right\}
$$

where $B_{1}$ and $B_{2}$ depend on $m$ and the choice of argument, $\beta=U_{c}^{\frac{1}{3}}, \alpha=\left(U_{c}^{\prime} P\right)^{\frac{1}{3}}$ and $\gamma$ is equal to $e^{1 \frac{\pi}{4}}$ or $e^{i \frac{5 \pi}{4}}$. When $\gamma=e^{i \frac{\pi}{4}}$, arg $\zeta$ must be taken in the sectors $\left\langle-\frac{3 \pi}{2},-\frac{\pi}{6}\right\rangle,\left\langle-\frac{\pi}{6}, \frac{7 \pi}{6}\right\rangle$, $\left\langle\frac{7 \pi}{6}, \frac{15 \pi}{6}\right\rangle$ for $\varphi^{(1,2,3)}$, respectively. The sectors must be turned an angle equal $-2 \pi$ for $\gamma=e^{i \frac{5 \pi}{4}}$.

The same method will be used to obtain asymptotic expansion of $\varphi^{(4)}, \varphi^{(5)}$ and $\varphi^{(6)}$. Ut1lizing

$$
M_{k, m}(z)=e^{-\frac{z}{2}} z^{m+\frac{1}{2}}(1+O(z)),
$$

we get

$$
\varphi^{(4,5,6)}(\zeta)=\text { const. } \times \zeta^{\frac{1}{2}-3 m}\left(1+0\left(\zeta^{-3}\right)\right) .
$$

(B7) is valid for arg $\zeta$ within the sectors $\left\langle-\frac{\pi}{6}, \frac{\pi}{2}\right\rangle,\left\langle-\frac{5 \pi}{6},-\frac{\pi}{6}\right\rangle$, $\left\langle-\frac{3 \pi}{2},-\frac{5 \pi}{6}\right\rangle$ for $\varphi^{(4,5,6)}$, respectiveiy.

C. The asymptotic validity of $\mathrm{w}_{1}$ and $\mathrm{w}_{2}$.

By using the connection formulae we will show that (B7) is available in greater sectors than stated. Concerning $\varphi^{(5)}$ we get from (A4) and (A7)

$$
\varphi^{(5)}=\varphi^{(4)}-\left(1+e^{i 6 m \pi}\right) \varphi^{(3)}
$$


and from $(A 4)$ and $(A 6)$

$$
\varphi^{(5)}=\varphi^{(6)}-\left(1+e^{i 6 \mathrm{~m} \pi}\right) \varphi^{(1)}
$$

$\varphi^{(3)}$ and $\varphi^{(1)}$ are exponentially decreasing for $-\frac{\pi}{2}<\arg \zeta<\frac{\pi}{6}$ and $-\frac{7 \pi}{6}<\arg \zeta<\frac{\pi}{2}$, respectively. The expansion of $\varphi^{(5)}$ in (B7) is therefore valid in the sector $-\frac{7 \pi}{6}<\arg \zeta<\frac{\pi}{6}$. Further,a Taylor expansion of (B7) about $s=0$ directly shows conformity with $w_{1}$ and $w_{2}$. Thus, from the above, $w_{1}$ and $w_{2}$ do provide valid asymptotic solutions of the disturbance equation for

$$
-\frac{7 \pi}{6}<\arg \left(z-z_{c}\right)<\frac{\pi}{6} .
$$

D. Some details.

The connection formulae can also be applied to extend the asymptotic validity of (B5) for $\varphi^{(1)}$ and $\varphi^{(3)}$. From (A7)

$$
\varphi^{(1)}(\zeta) \sim-\varphi^{(2)}(\zeta), \text { for }-\frac{\pi}{6}<\arg <\frac{\pi}{6}
$$

and from (A8)

$$
\varphi^{(3)}(\zeta) \sim-\varphi^{(2)}(\zeta) \text {, for }-\frac{7 \pi}{6}<\arg \zeta<-\frac{5 \pi}{6}
$$

The matter is that $\varphi^{(2)}(\zeta)$ is exponentially increasing for arg $\zeta$ within the above two sectors.

In view of the present characteristic problem it is convenient to introduce the transformation

$$
t=U_{c}^{\frac{1}{3}} \tau \quad \text { and } \quad y=-\left.U_{c}^{\frac{1}{3}} \zeta\right|_{z=-1}
$$

Applying (D3) in (A1) will do the integral independent of $c$. 


\section{$\underline{\text { References }}$}

[1] Heisenberg, W., Über Stabilität und Turbulenz von Flüssigkeitsströmen, Ann.d.Phys. 74, S.577-627 (1924).

[2] Tollmien, W., Über die Entstehung der Turbulenz, Nachr.v.d. Ges.d.Wiss. zu Göttingen, Math.-Phys.Klasse, $1, S .21-44$ (1929).

[3] Schlichting, H.,Amplitudenverteilung und Energiebilanz der kleinen Störungen bei der Plattenströmung, Nachr.v.d.Ges.d.Wiss. zu Göttingen, Math.1, S.47-78 (1935).

[4] Iin, C.C., On the stability of two-dimensional parallel flows, Parts I, II, III, Quart.Appl.Math. 3, pp.117-142, 218-234, 277-301 (1945).

[5] Koppel, D., On the stability of flow of a thermally stratified fluid under the action of gravity, J.Math. Phys. 5, pp.963-982 (1964).

[6] Gage, K.S. and Reid, W.H., The stability of thermally stratified plane Poiseulle flow, J.Fluid Mech. 33, pp.21-32 (1958).

[7] Hughes, T.H. and Reid, W.H., The stability of spiral flow between rotating cylinders. Phil. Trans.,Roy.Soc. of London, 263A, pp.57-91 (1968).

[8] Erdélyi, A. and Swanson, C.A., Asymptotic forms of Whittaker's confluent hypergeometric functions, Memoirs of the American Math.Soc. 25, pp. 1-49 (1957). 


\section{Figure legends}

Figure 4.1. Neutral stability curves in the $(k, R)$-plane. Points inside each curve correspond to unstable conditions.

Figure 4.2. Neutral stability curves in the (c,k)-plane. Points inside each curve correspond to unstable conditions.

Figure 5.1. Neutral stability curves in the (Ra,R)-plane. Points below each curve correspond to stable conditions for $R^{\frac{1}{3}}<(5400)^{\frac{1}{3}} \approx 17.5$, and to unstable conditions for $\mathrm{R}^{\frac{1}{3}}>17.5$.

Figure A1. The paths of integration in the $t$-plane for Koppel's solutions. 


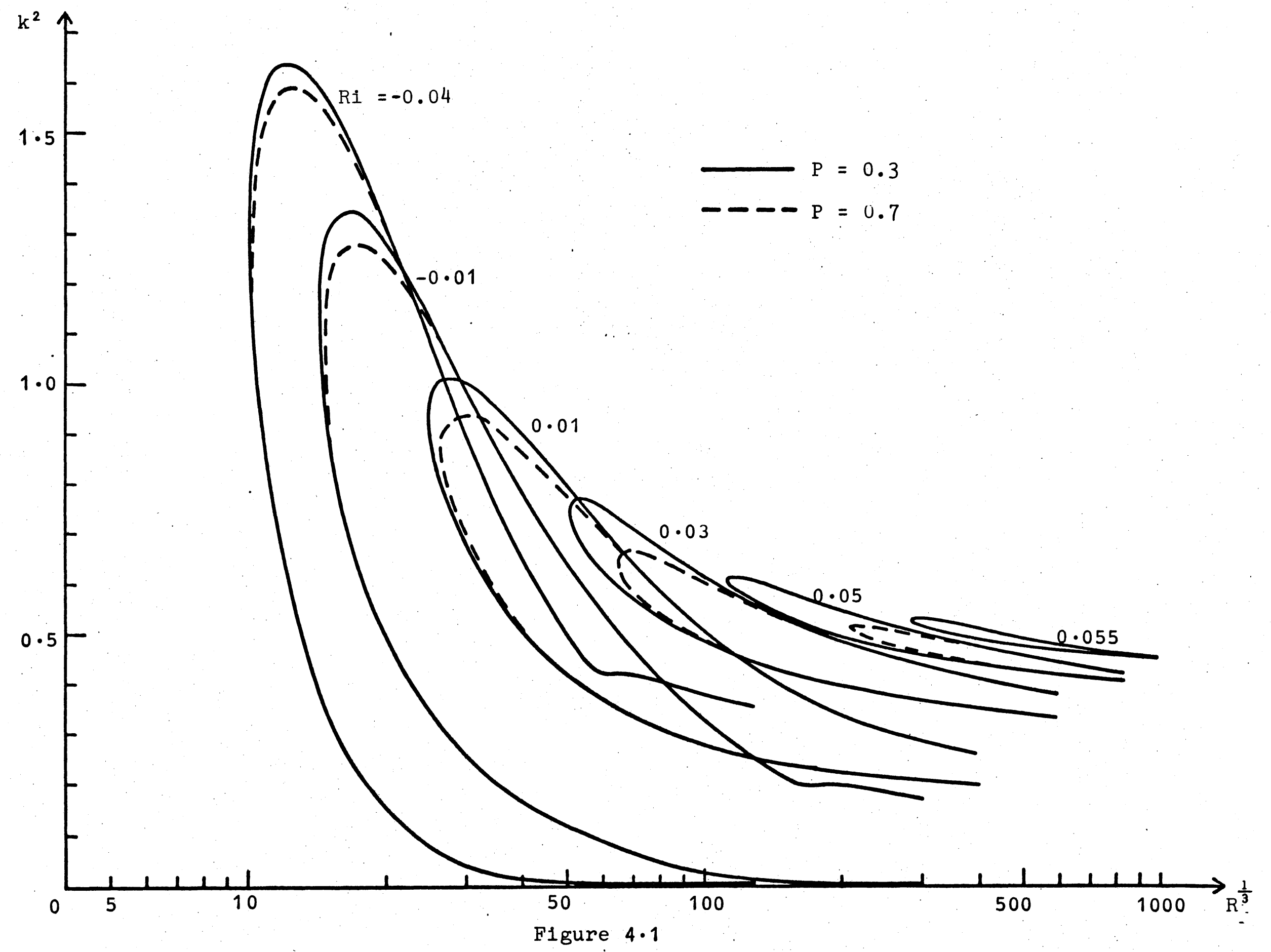




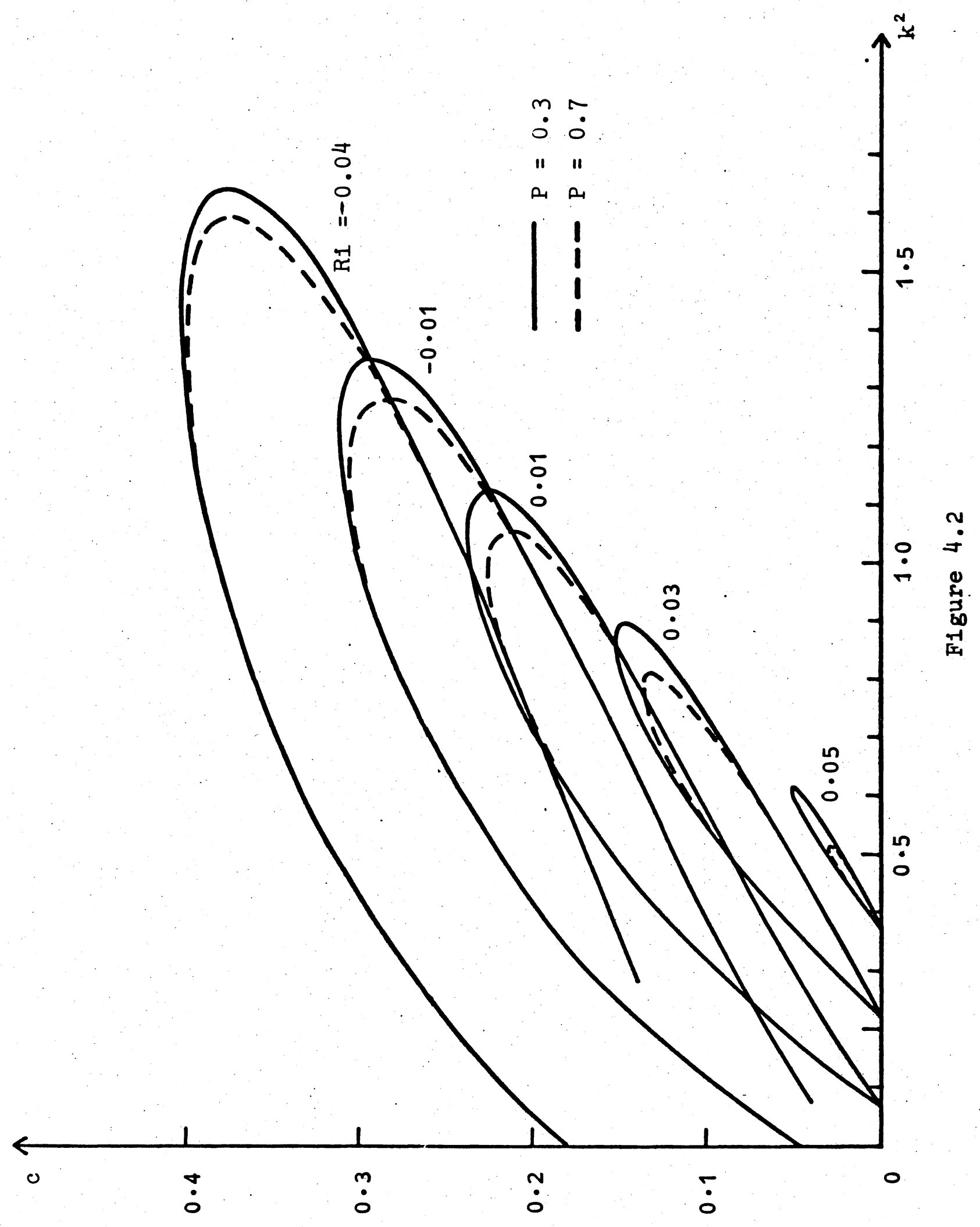




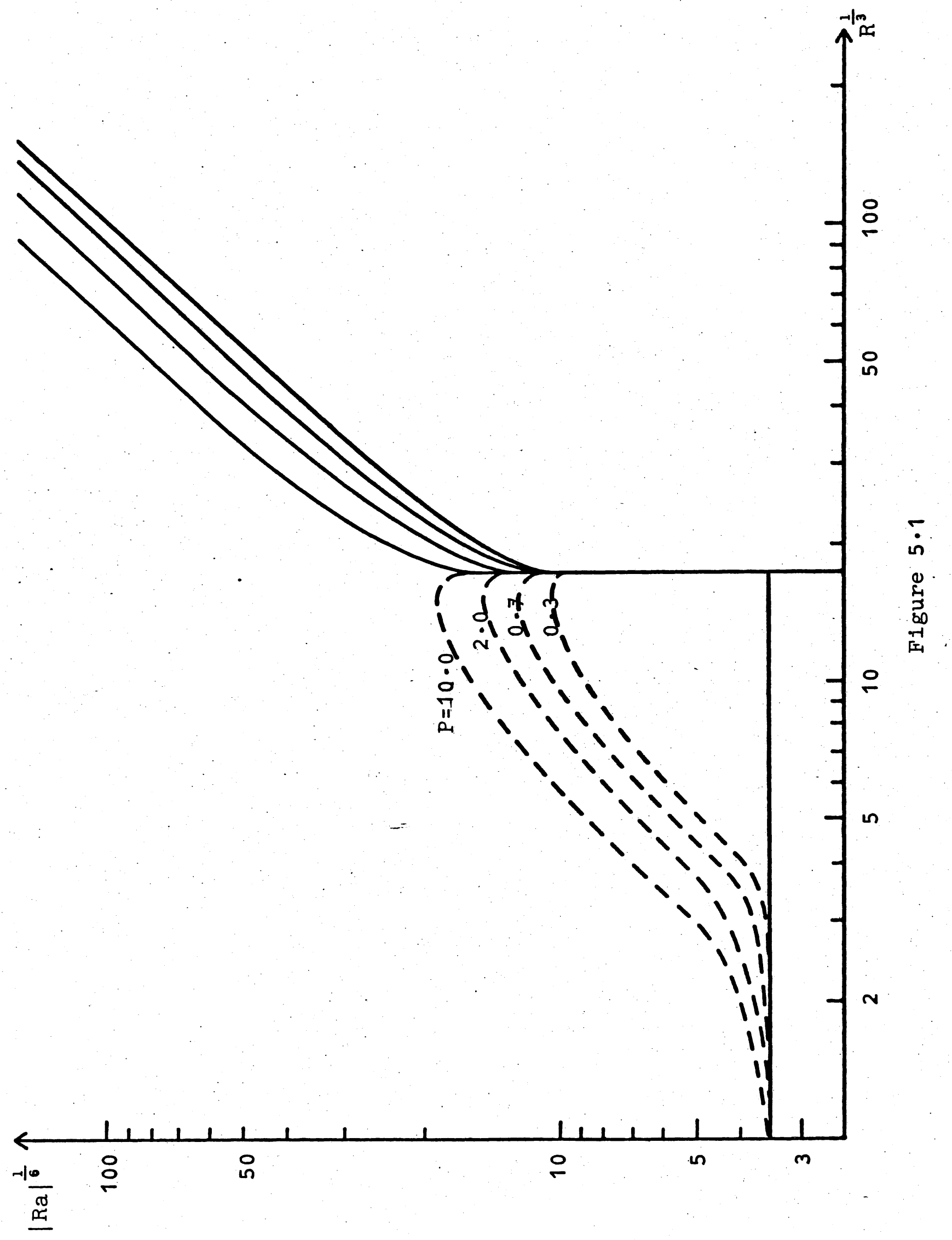



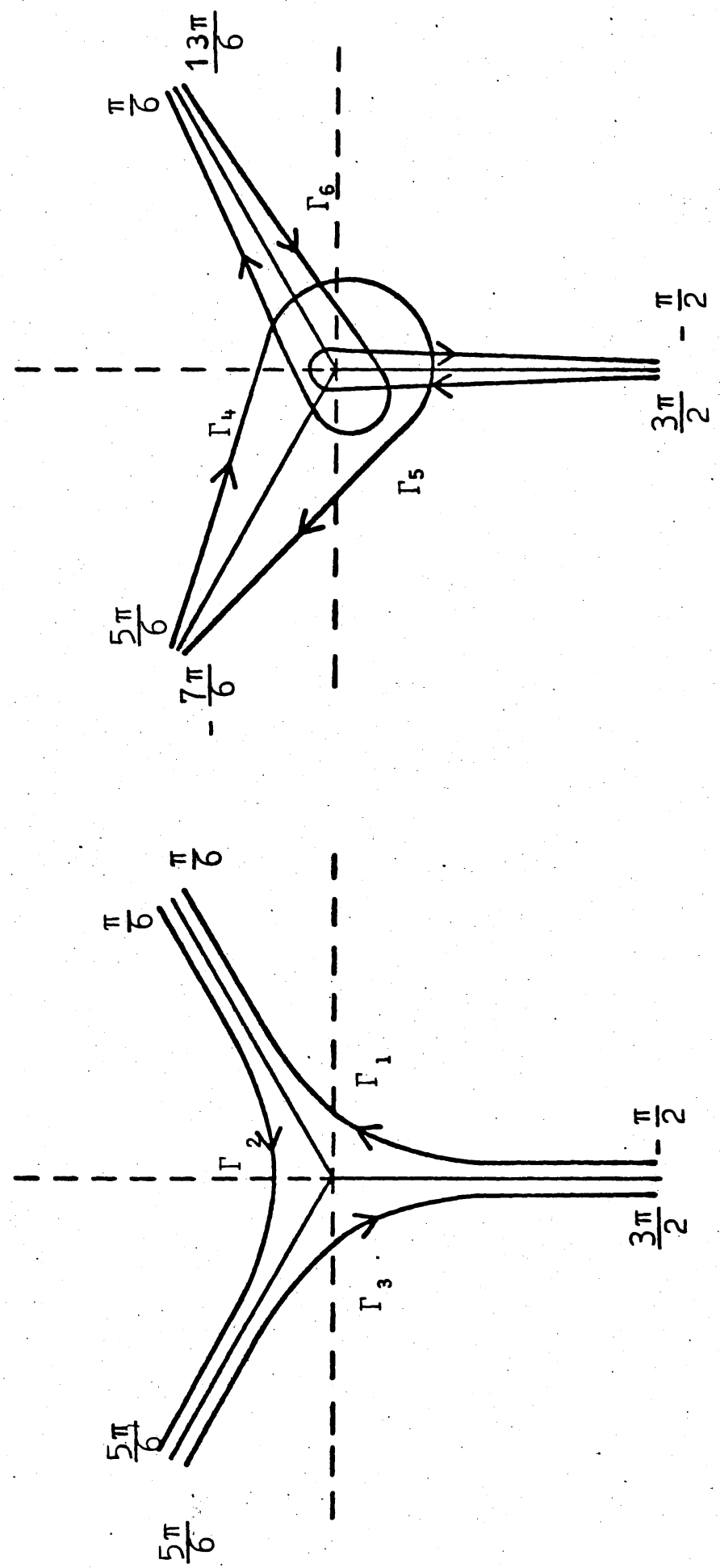

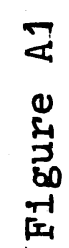

